\title{
Introducing reactive modal tableaux
}

\author{
Dov Gabbay
}

Published online: 29 September 2012

(C) Springer Science+Business Media B.V. 2012

\begin{abstract}
This paper introduces the idea of reactive semantics and reactive Beth tableaux for modal logic and quotes some of its applications. The reactive idea is very simple. Given a system with states and the possibility of transitions moving from one state to another, we can naturally imagine a path beginning at an initial state and moving along the path following allowed transitions. If our starting point is $s_{0}$, and the path is $s_{0}, s_{1}, \ldots, s_{n}$, then the system is ordinary non-reactive system if the options available at $s_{n}$ (i.e., which states $t$ we can go to from $s_{n}$ ) do not depend on the path $s_{0}, \ldots, s_{n}$ (i.e., do not depend on how we got to $s_{n}$ ). Otherwise if there is such dependence then the system is reactive. It seems that the simple idea of taking existing systems and turning them reactive in certain ways, has many new applications. The purpose of this paper is to introduce reactive tableaux in particular and illustrate and present some of the applications of reactivity in general. Mathematically one can take a reactive system and turn it into an ordinary system by taking the paths as our new states. This is true but from the point of view of applications there is serious loss of information here as the applicability of the reactive system comes from the way the change occurs along the path. In any specific application, the states have meaning, the transitions have meaning and the paths have
\end{abstract}

D. Gabbay

Bar Ilan University, Ramat-Gan, 52900, Israel

D. Gabbay $(\bowtie)$

King's College London, London, UK

e-mail: dov.gabbay@kcl.ac.uk

D. Gabbay

University of Luxembourg, Luxembourg, Belgium 
meaning. Therefore the changes in the system as we go along a path can have very important meaning in the context, which enhances the usability of the model.

Keywords Modal logic • Other nonclassical logic • Combined logics • Logic in computer science $\cdot$ Beth tableaux

Mathematics Subject Classifications (2010) $03 \mathrm{~B} 45 \cdot 03 \mathrm{~F} 03$

\section{Setting the scene}

The purpose of this section is to explain the idea of reactive semantics and reactive Beth tableaux. The best way to do it is to begin with an ordinary (non-reactive) example of a modal logic, look at its axioms and semantics and give the corresponding ordinary (non-reactive) Beth tableaux for it. We then proceed to turn the example reactive and see what kind of modifications we need to do to the Beth tableaux we have developed. These modifications will introduce us to our first example of reactive Beth tableaux. For general references on tableaux and on modal tableaux, see [1, 3-5, 20]. For general papers on Reactivity and its use see [2, 6, 7, 10, 11, 13-19].

This will be done in the first section. The second section defines the general notion of Reactive Beth tableaux and the third section discusses our options for reactivity in general, including reactive Beth tableaux, reactive automata, reactive sets and more.

Let us begin with modal logic. We denote a Kripke frame by $\mathbf{F}=(S, R, t)$ where $S \neq \varnothing$ is the set of possible worlds and $R \subseteq S \times S$ is the accessibility relation and $t \in S$ is the actual world.

Our language contains the set of atoms $Q$ and the usual connectives $\{\top, \perp, \sim$ $\wedge, \vee, \diamond, \rightarrow$ and $\square$ \}.

An assignment $h$ into a frame $\mathbf{F}=(S, R, t)$ is a function giving to each $q \in Q$ a subset $h(q) \subseteq S$. A Kripke model has the form $\mathbf{m}=(S, R, t, h)=(\mathbf{F}, h)$. We evaluate a wff in a model as in Definition 1.1 below. We call it the ordinary evaluation (as opposed to the reactive evaluation, which we shall describe later).

Definition 1.1 (Ordinary evaluation in Kripke models) We write

$$
s \vDash A \text { in } \mathbf{m},
$$

to mean

A holds at $s$ in the model $\mathbf{m}=(S, R, t, h)$.

The following are the clauses (we omit $\mathbf{m}, h$ since the model does not change).

1. $s \vDash q$ iff $s \in h(q)$, for $q$ atomic.

- $s \vDash A \wedge B$ iff $s \vDash A$ and $s \vDash B$.

- $S \models \sim A$ iff $s \not \models A$.

- The cases of $A \vee B, A \rightarrow B$ are defined in the standard way from $\sim$ and $\wedge$.

2. $s \vDash \square A$ iff for all $x$ such that $s R x$ we have $x \vDash A$.

3. We say that $\mathbf{m}=(S, R, t, h)$ satisfies $A$ (we write $\mathbf{m} \vDash A$ ) iff $t \vDash A$. 
4. We say that $A$ holds in the frame $(S, R, t)$ iff for all possible assignments $h$ into the frame we have $(S, R, t, h) \vDash A$.

5. Let $\mathbb{K}$ be a class of frames and let $\mathbb{L}(\mathbb{K})$ be the set of all sentences which hold in every frame of $\mathbb{K}$. We say that $\mathbb{L}(\mathbb{K})$ is the modal logic defined by $\mathbb{K}$.

There are several ways of characterising the logic $\mathbb{L}(\mathbb{K})$ of Definition 1.1.

1. We can generate $\mathbb{L}(\mathbb{K})$ by giving a Hilbert or Gentzen or natural deduction system $\mathbf{S}$ for the language, and define the notion of $A \vdash_{\mathbf{S}} B$ which means that $B$ is provable in the system $\mathbf{S}$ from $A$. This is an algorithmic notion defined using $\mathbf{S}$.. The completeness theorem would mean the following:

- $A \vdash_{\mathbf{S}} B$ iff for every model $\mathbf{m}$ of $\mathbb{K}$ we have that $\mathbf{m} \vDash A$ implies $\mathbf{m} \vDash B$.

2. We can present a tableaux system for $\mathbb{L}(\mathbb{K})$ which tries to build a countermodel for any pair $A \vdash ? B$, showing, if successful, a model $\mathbf{m}$ with frame in $\mathbb{K}$ such that $\mathbf{m} \vDash A$ but $\mathbf{m} \not \models B$.

The tableaux system is an algorithm which constructs (if possible) a countermodel by following closely the options allowed by the semantics $\mathbb{K}$.

The completeness theorem would mean the following:

- The tableau construction for $A \vdash ? B$ is successful iff there exists a model m of $\mathbb{K}$ in which $A$ holds and $B$ does not hold.

It is not easy to find a proof system $\mathbf{S}$ or a tableaux system for arbitrary $\mathbb{K}$. It is easier to find a tableaux system for a logic defined by a single frame. So this is what we discuss now and we choose a single extremely simple frame to illustrate our reactive ideas.

\subsection{First case study}

From the point of view of modal logic, classical logic is the logic of one point Kripke frame $\mathbf{F}=(S, R, t)$ with a reflexive accessibility relation.

We want to explain the idea of reactive Beth tableaux using this modal logic. So consider the modal logic with one point frame $\mathbf{F}_{0}$ of Fig. 1.

In this logic we have

$$
\vDash A \leftrightarrow \diamond A .
$$

It is easy to put forward an axiom system for $\mathbb{L}\left(\mathbf{F}_{0}\right)$. We take any axiom system for classical logic and add the axiom $A \leftrightarrow \diamond A$.

The tableaux for this logic is clear. We use the tableaux and rules for classical logic together with two additional rules for $\diamond$ which identify $\diamond A$ with $A$. Since we

Fig. 1 Frame $\mathbf{F}_{0}$

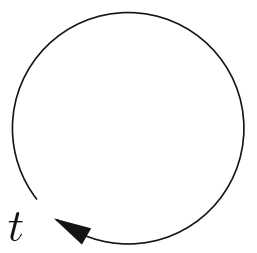


are going to turn this logic reactive, and formulate reactive tableaux for it, let us write the familiar tableau rules explicitly in a way which makes it ready for becoming reactive.

\section{Definition 1.2 (Tableaux for $\mathbf{F}_{0}$ )}

1. A tableau $\tau$ is a two compartment box with a label. The compartments contain formulas of the logic. The left compartment contains formulas intended to be true (hold) and the right compartment contains formulas intended to be false (not to hold). The label of the tableau contains information about the tableau including which possible world we are talking about, as well as additional information to be used when we turn the tableau reactive.

Figure 2 shows an example of a tableau $\tau$. The label is $s$ and the formulas are $A_{i}$ and $B_{j}, i=1, \ldots, n, j=1, \ldots, k$.

2. A tableaux is said to be closed if the same formula $A$ appears in both the right and left box, or if $\perp$ appears in the left box or if $T$ appears in the right box.

A tableaux is said to be a world (or a model) if all atoms of the language appear in it, and it is not closed.

3. We consider a system of tableaux as a set $\tau$ of tableaux. The system is closed if all tableaux in the system are closed.

Definition 1.3 (Operations on a set $\tau$ of tableaux) We start with a set $\tau$ containing a single tableau as in Fig. 2 where $s$ is the actual world $t$. We perform successive operations on the elements of $\boldsymbol{\tau}$ to obtain successive sets of tableaux $\boldsymbol{\tau}_{1}, \boldsymbol{\tau}_{2}, \boldsymbol{\tau}_{3} \ldots$ Each time we perform an operation on $\tau_{n}$, we choose a tableaux $\tau \in \tau_{n}$ and apply a rule on $\tau$ to obtain from it one or two simpler tableaux $\tau^{\prime}, \tau^{\prime \prime}$ and then we let $\boldsymbol{\tau}_{n+1}=$ $\left(\boldsymbol{\tau}_{n}-\{\tau\}\right) \bigcup\left\{\tau^{\prime}, \tau^{\prime \prime}\right\}$. This way we keep on making the tableaux in $\boldsymbol{\tau}_{n}$ more and more simple until no more rules apply. Say we stop and cannot continue at stage $\boldsymbol{\tau}_{m}$. At this stage all the tableaux in $\boldsymbol{\tau}_{m}$ contain only atoms in their respective boxes (this will become apparent by looking at the rules below). We will get that if all tableaux in $\boldsymbol{\tau}_{m}$ are closed then there is no model which makes all formulas $A_{i}$ true and all $B_{j}$ false (see Fig. 2).

The operations are done by choosing a tableau $t \in \boldsymbol{\tau}$ for example:

$$
\tau=t_{1}: \begin{array}{|l|l|}
\hline A_{i} & B_{j} \\
\hline
\end{array}
$$

and choosing a formula $A$ in the left or right box and operating on it using the tableaux rules.

The following are the rules for $\wedge$ and $\sim$ on the left and on the right. Our set of labels is $\{t\}$ with only one label. However, since we are going to turn the tableaux

Fig. 2 Tableaux $\tau$

$$
\begin{array}{ll|l}
\multicolumn{1}{l}{} & \multicolumn{2}{l}{\text { Left }} \\
\cline { 2 - 3 } & A_{1}, \ldots, A_{n} & B_{1}, \ldots, B_{k} \\
\hline
\end{array}
$$


rules reactive we write the rules schematically as if more labels were available at play.

\section{Left $\sim$ rule for label $t_{1}$}

Replace

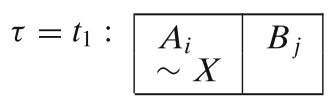

by

$$
\tau_{1}=t_{2}: \begin{array}{|l|l|}
A_{i} & B_{j} \\
& X \\
\hline
\end{array}
$$

where $t_{2}=t_{1}$.

Right $\sim$ rule for label $t_{1}$

Replace

$$
\tau=t_{1}: \begin{array}{l|l|}
A_{i} & \begin{array}{l}
B_{j} \\
\sim X
\end{array} \\
\hline
\end{array}
$$

by

$$
\tau_{1}=t_{2}: \begin{array}{|c|c|}
\hline A_{i} & B_{j} \\
X & \\
\hline
\end{array}
$$

where $t_{2}=t_{1}$.

\section{Left $\wedge$ rule for label $t_{1}$}

Replace

$$
\tau=t_{1}: \begin{array}{|l|l|}
\hline A_{i} & B_{j} \\
X \wedge Y & \\
\hline
\end{array}
$$

by the tableau

$$
\tau_{1}=t_{2}: \begin{array}{|l|l|}
\hline A_{i} & B_{j} \\
X, Y & \\
\hline
\end{array}
$$

where $t_{2}=t_{1}$.

\section{Right $\wedge$ rule for label $t_{1}$}

Replace

$$
\tau=t_{1}: \begin{array}{|l|l|}
\hline A_{i} & B_{j} \\
& X \wedge Y \\
\hline
\end{array}
$$


by the tableaux $\tau_{1}$ and $\tau_{2}$

$$
\begin{aligned}
& \tau_{1}=t_{2}: \begin{array}{|l|l|}
\hline A_{i} & B_{j} \\
& X \\
\hline
\end{array} \\
& \tau_{2}=t_{2}: \begin{array}{l|l|}
A_{i} & B_{j} \\
& Y \\
\hline
\end{array}
\end{aligned}
$$

where $t_{2}=t_{1}$.

Notice that the label $t_{1}$ has not changed after the operations, we always have $t_{2}=t_{1}$.

We need not explicitly give the rules for $\vee$ and $\rightarrow$ as $\sim, \wedge$ are sufficient for classical logic and anyway, the rules for $\vee$ and $\rightarrow$ are well known, and the reader can easily figure them out in our notation.

For the logic with $\diamond$, we add the following rules:

\section{Left $\diamond$ rule for label $t_{1}$}

Replace

$$
\tau=t_{1}: \begin{array}{|l|l|}
\hline A_{i} & B_{j} \\
\diamond X & \\
\hline
\end{array}
$$

by

$$
\tau_{1}=t_{2}: \begin{array}{|c|c|}
\hline A_{i} & B_{j} \\
X & \\
\hline
\end{array}
$$

where $t_{2}=t_{1}$.

\section{Right $\diamond$ rule for label $t_{1}$}

Replace

$$
\tau=t_{1}: \begin{array}{l|l|}
A_{i} & B_{j} \\
& \diamond X \\
\hline
\end{array}
$$

by

$$
\tau_{1}=t_{2}: \begin{array}{|l|l|}
\hline A_{i} & B_{j} \\
& X \\
\hline
\end{array}
$$

where $t_{2}=t_{1}$.

Theorem 1.4 (Completeness theorem) Consider the logic for the frame $\mathbb{F}_{0}$ of Fig. 1 , and let $A$ and $B$ be two formulas. Then $A \vDash B$ iff the tableaux

$$
\boldsymbol{\tau}_{0}=\left\{t: \begin{array}{|l|l|}
\hline A & B
\end{array}\right\}
$$

can be expanded into a closed system of tableaux by means of the reactive tableaux rules. 
Proof Well known process, same as for classical logic. We use the additional axiom $\diamond A \leftrightarrow A$ in the right and left rules for $\diamond$ which basically eliminate $\diamond$.

We now turn our system reactive. Consider the frame $\mathbb{F}_{1}$ of Fig. 3

The set is $S=\{t\}$. The relation of accessibility is $\mathbb{R}=\{(t, t),((t, t)(t, t))\}$. $(t, t)$ is the arrow and $((t, t),(t, t))$ is the double arrow. The reactivity idea is expressed by the point of view that once we pass through the arrow, this activates the double arrow which disconnects the reflexive arrow. We are not giving a formal definition at this stage, instead we give an example. The formal definitions are Definitions 1.8 and 1.9.

Example 1.5 (Reactive evaluation in $\mathbb{F}_{1}$ ) Let $q$ be an atom and assume $t \vDash q$.

To evaluate $t \vDash ? \diamond q$ we go to the accessible world $t$ ( $t \mathbb{R} t$ holds) and evaluate $q$. Since $t \vDash q$, we get that $t \vDash \diamond q$.

Now let us evaluate $t \vDash ? \diamond \diamond q$. We go to the accessible world $t$ and evaluate $t \vDash ? \diamond q$. But now, having passed through the arrow $(t, t)$ the double arrow $((t, t),(t, t))$ was activated, and disconnected the reflexive arrow $(t, t)$. The model has changed. What we have now is a model with the frame $(\{t\}, \varnothing, t\})$. Hence now $t \vDash \diamond q$ is false. Thus

$$
t \not \forall \diamond \diamond q
$$

It is obvious that in a reactive model in general the value of $s \models$ ? $\diamond A$ depends not just on $s$ but on the past history sequence of points of evaluations $\left(s_{1}, \ldots, s_{k}, s\right)$ leading to $s$. So we must use the notation

$$
\left(s_{1}, \ldots, s_{k}, s\right) \vDash ? \diamond A .
$$

The sequence of points leading to $s$ will activate various reactivity double arrows and tell us what is the current accessibility situation at the point $s$.

Definition 1.6 (Reactive satisfaction in $\mathbb{F}_{1}$ ) The following is the correct way of defining evaluation in our reactive model of Fig. 3. We have paths $t, t t, t t$, and we give evaluation clauses for each path. We need use only two labels, $t$ and $t t$, because additional $t$ 's make no behavioural difference:

- $\quad t \vDash q$ if $t \in h(q), q$ atomic

- $t t \vDash q$ if $t \in h(q), q$ atomic

- The evaluation at $t, t$ of $\wedge$ and $\sim$ is as in classical logic.

- $\quad t \vDash \diamond A$ iff $t t \vDash A$

- $t t \not \forall \diamond A$

- $A$ holds in the modal if $t \vDash A$.

Fig. 3 Frame $\mathbb{F} 1$

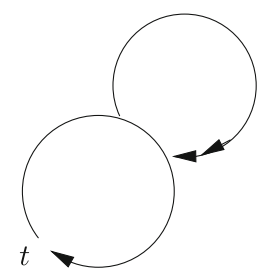


We now check what kind of logic we get from the satisfaction notion of Definition 1.6. Note that the notion of "logic" here is slightly different. These logics are not closed under arbitrary substitution. You can see this from the way we axiomatise our examples, see Definition 1.11.

Theorem 1.7 The logic of the frame of Fig. 3 as defined by the satisfaction notion of Definition 1.6 is not complete for any class of traditional Kripke frames

Proof Let us assume that the logic of the figure is complete for a class of Kripke frames. We get a contradiction. Since the formula $q \wedge \sim \diamond \diamond q$ is consistent in the logic of the figure, it must have a model. Let $\mathbb{K}$ be a class of traditional Kripke frames for which the logic is complete and let $(S, R, t, h)$ be a model of $q \wedge \sim \diamond \diamond q$. The frame $(S, R, t)$ must be a model of the logic under any assignment $h$ to the frame.

Claim 1 tRt must hold.

Otherwise, let $p$ be atomic and let $t \vDash p$, but let $s \not \models p$ for any $s \neq t$. Hence $t \vDash$ $p \wedge \sim \diamond p$.

This is impossible since in the reactive frame of Fig. 3 we have $\vDash p \rightarrow \diamond p$, for any atomic $p$, according to Definition 1.6.

Claim $2 q \rightarrow \diamond \diamond q$ holds under $h$.

This holds because $t R t$ holds.

We now have a contradiction.

It is time we define the notion of switch reactive Kripke models. Section 3 will discuss other options for reactivity in Kripke models. The switch reactivity is the simplest.

Definition 1.8 (Switch reactive models)

1. A switch reactive Kripke frame has the form $(S, \mathbb{R}, \mathbf{a}, t)$ where $S$ is a non-empty set of possible worlds, $t \in S$ is the actual world and $\mathbb{R}$ is a reactive accessibility set of arrows on $S$ defined as follows:

(a) $(t, s) \in S \times S$ is an arrow of level 1 .

(b) If $\alpha$ is an arrow of level $n$ and $(t, s) \in S \times S$ then $((t, s), \alpha)$ is an arrow of level $n+1 .^{1}$

${ }^{1}$ An alternative way of defining $\mathbb{R}$ is as follows:

A $k$-level arrow is inductively defined as follows:

$$
\begin{aligned}
& \mathbb{A}_{0}=S \times S \\
& \mathbb{A}_{k+1}=(S \times S) \times \mathbb{A}_{k} .
\end{aligned}
$$

Let $\mathbb{A}=\bigcup \mathbb{A}_{k}$.

Let $\mathbb{R}$ be a subset of $\mathbb{A}$. 
We assume that if $((t, s), \alpha) \in \mathbb{R}$ then $\alpha \in \mathbb{R}$.

a is a $\{0,1\}$ function on $\mathbb{R}$.

If $\mathbf{a}(\alpha)=1$, we say $\alpha$ is active.

$t$ is the actual world.

If $\mathbf{a}(\alpha)=0$, we say $\alpha$ is not active.

2. Let $(t, s) \in \mathbb{R}$. Define $\mathbf{a}_{(t, s)}$ as follows

$$
\begin{gathered}
\mathbf{a}_{(t, s)}(\beta)=1-\mathbf{a}(\beta), \text { if }((t, s), \beta) \in \mathbb{R} \\
\mathbf{a}_{(t, s)}(\beta)=\mathbf{a}(\beta) \text { otherwise. }
\end{gathered}
$$

Definition 1.9 (Satisfaction in switch reactive model) Let $\mathbf{m}=(S, \mathbb{R}, \mathbf{a}, t, h)$ be a reactive switch model where $(S, \mathbb{R}, \mathbf{a}, t)$ is a switch reactive frame and $h$ is an assignment into $S$ giving for each atomic $q$ a subset $h(q) \subseteq S$. We define the notion of $\mathbf{m} \vDash A$.

1. $\mathbf{m} \vDash q$ iff $t \in h(q)$

2. $\mathbf{m} \vDash A \wedge B$ iff $\mathbf{m} \vDash A$ and $\mathbf{m} \vDash B$

$\mathbf{m} \vDash \sim A$ iff $\mathbf{m} \not \models A$

similarly for $\wedge, \vee, \perp, \top$.

3. $\mathbf{m} \vDash \diamond A$ iff for some $(t, s) \in \mathbb{R}$ such that $\mathbf{a}((t, s))=1$ we have

$$
\mathbf{n}=\left(S, \mathbb{R}, \mathbf{a}_{(t, s)}, s, h\right) \vDash A
$$

4. Note that the real model is the active part of $\mathbb{R}$. When we evaluate $\diamond$ we move to a new model with different active part.

5. Given a frame $\mathbb{F}=(S, \mathbb{R}, \mathbf{a}, t)$ then the logic of the frame $\mathbb{L}(\mathbb{F})$ is the set of all wff $A$ such that for all $h(S, \mathbb{R}, \mathbf{a}, t, h) \vDash A$.

Corollary 1.10 There exists a logic complete for a class of reactive frames but not complete for a class of ordinary frames.

Definition 1.11 (Axiomatisiation of the logic of the reactive frame of Fig. 3) We offer the following system:

1. Take as axioms the following:

(*) All substitution instances of truth functional tautologies $A\left(q_{1}, \ldots, q_{k}\right)$.

2. Let $A\left(q_{1}, \ldots, q_{k}\right)$ be a wff. $A$ can be presented as a substitution result of the form

$$
A\left(q_{1}, \ldots, q_{k}\right)=B\left(q_{1}, \ldots, q_{k}, e_{i} / \diamond B_{i}\right)
$$

$i=1, \ldots, m$, where $e_{i}$ are new atoms and $B_{i}$ are formulas using the atoms $q_{1}, \ldots, q_{k}$ and $B\left(q_{1}, \ldots, q_{k}, e_{1}, \ldots, e_{m}\right)$ is a formula without a modality. 
This representation is unique.

Let $B_{A}^{\perp}$ be the formula $B\left(q_{1}, \ldots, q_{k}, \perp, \ldots, \perp\right)$.

We take the additional following axiom:

(**) $\diamond A$ for any $A$ such that $B_{A}^{\perp}$ is a truth functional tautology.

Example 1.12 This example prepares the ground for giving a reactive tableaux algorithm for the logic of Fig. 3. Although this logic is very simple, there is a technical problem with the reactive tableaux for it. This is typical of all reactive tableaux and arises from the very idea of reactivity. Consider Fig. 3 and let $p, q$ be two atoms such that $t \vDash p \wedge q$. Consider now $t \vDash ? \diamond p \wedge \diamond q$.

According to the definition of satisfaction, the evaluation is done in parallel.

$$
t \vDash \diamond p \wedge \diamond q
$$

iff

$$
t \vDash \diamond p \wedge t \vDash \diamond q
$$

iff

$$
t t \vDash p \wedge t t \vDash q
$$

When we give any tableaux algorithm the algorithm is sequential.

So if we apply a tableaux rule corresponding to $t \vDash \diamond p$, we go to $t t$ in the model and in the corresponding tableau and have $p, \diamond q$ to evaluate and in $t t, \diamond q$ is false!

Put differently, if we evaluate $\diamond p$ first and pass through some arc to find a world in which $p$ holds, the model might change. We should backtrack on the change when we evaluate $\diamond q$.

So the tableaux need to be done in parallel.

Definition 1.13 (Tableaux system for the logic of the frame of Fig. 3) We modify the tableaux system of Definition 1.11 as follows:

1. We allow for two labels $t$ and $t$.

2. We require that all classical connective in each box be handled first before approaching any $\diamond$. For that purpose we regard any $\diamond X$ formula as 'atomic' and bring each tableaux to its 'atomic' components. The reason for that has to do with Example 1.12 and the need to handle all cases of $\diamond$ all in parallel.

3. The rules for $\sim$ and $\wedge$ remain the same as in Definition 1.3, where $t_{1}$ can now be either $t_{1}=t$ or $t_{1}=t t . t_{2}$ remains equal to $t_{1}$.

4. The rules for $\diamond$ now split into two sets. For the case of $t_{1}=t$, we take the rules of Definition 1.3 with $t_{2}=t t$ (and not $t_{2}=t_{1}$ as in Definition 1.3).

We have a condition here that all cases of $\diamond X, \diamond Y$ etc both on the right hand side or on the left hand side should all be done in parallel. The classical connectives should be dealt with before $\diamond$ is handled. 
For the case of $t_{1}=t t$ we take the following rules:

\section{Left $\diamond$ rule label $t t$}

Replace

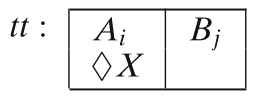

by

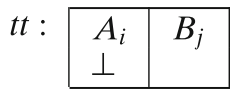

Right $\diamond$ rule for label $t t$

Replace

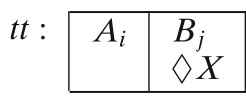

by

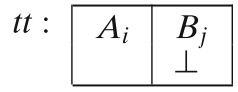

Theorem 1.14 The tableaux system of Definition 1.13 is complete for the semantics of $\mathbb{F}_{2}$ and for the axiomatisation of Definition 1.11.

Proof Not too difficult.

Remark 1.15 The example we gave is rather simple. In Section 1.2 we give a slightly more complex example.

\subsection{Second case study}

We now consider another example. Consider frame $\mathbf{F}_{2}$ of Fig. 4.

We have $S=\{t, s\}$ and $R=\{(t, t),(t, s),(s, t)\}$. 
Fig. 4 Frame $\mathbf{F}_{2}$

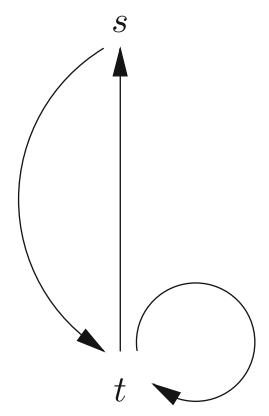

As we have already said, there are several ways to characterise the logic of this frame.

1. Write a Hilbert or a Gentezen system for it and get a completeness theorem for it, namely:

$A \vdash_{\text {system }} B$ iff for all assignments $h$ to the frame $\mathbf{F}_{2}$ above we have $t \vDash_{h} A$ implies $t \vDash_{h} B$.

2. Provide a tableaux system for it and have the following completeness theorem

$A \nvdash_{\text {system }} B$ iff there is successful tableaux procedure for $A=\top, B=\perp$.

The fact that we are dealing with the frame $\mathbf{F}_{2}$ of Fig. 4 would be incorporated in the tableaux procedures.

We now present a reactive version of Fig. 4. Consider Fig. 5, displaying the frame $\mathbb{M}$

In this figure we added a double arrow from the arc $(t, t)$ into itself. We can mathematically present the frame as $\mathbb{M}=(S, \mathbb{R}, t)$ where $S=\{t, s\}$ and $\mathbb{R}=$ $\{(t, t),(t, s),(s, t),((t, t),(t, t))\}$.

The item $((t, t),(t, t))$ represents the double arrow. The evaluation of a formula at $t$ under an assignment $h$ to the atoms goes as in Definition 1.16 below. Here we use the full notation in the index:

Definition 1.16 (Reactive evaluation in the frame $\mathbb{M}$ ) Let $x \in\{t, s\}$ :

1. - $x \vDash_{h} q$ iff $x \in h(q)$, for $q$ atomic.

- $x \vDash_{h} A \wedge B$ iff $x \vDash A$ and $x \vDash B$.

- $\quad x \vDash_{h} \sim A$ iff $x \not \models A$.

Fig. 5 Frame $\mathbb{M}$

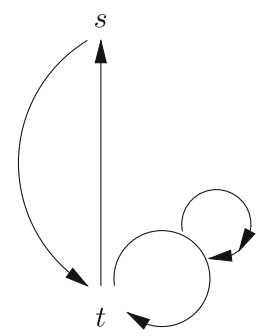


Fig. 6 Frame $\mathbf{N}$

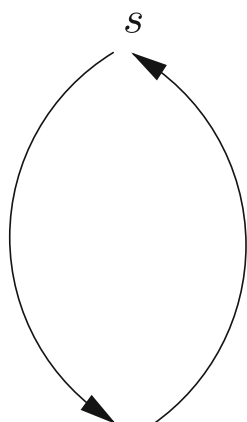

$t$

2. $t \vDash_{h} \square A$ iff (a) and (b) hold.

(a) $s \vDash_{h} A$, in the frame $\mathbb{M}=(S, \mathbb{R}, t)$.

(b) $t \models_{h} A$ in the frame $\mathbf{N}=\left(S, R_{1}, t\right)$ where $S=\{t, s\}$ and $R_{1}=\{(t, s),(s, t)\}$. See Fig. 6.

3. $s \vDash_{h} \square A$ in $\mathbb{M}$ or $\mathbf{N}$ iff $t \vDash_{h} A$ in $\mathbb{M}$ or $\mathbf{N}$ respectively.

The way we view the change of model is as follows.

To evaluate $t \vDash \square A$, we need to go to all accessible worlds from $t$. These are in our frame $\mathbb{M}$, the worlds $t$ and $s$. To go to $s$ we traverse the $\operatorname{arc}(t, s)$ and we get to $s$ and at $s A$ must hold. We thus get condition (a) above. We also go to $t$ along the $\operatorname{arc}(t, t)$. As we traverse the arc $(t, t)$, we activate the double arrow $((t, t),(t, t))$ which reactively disconnects the arc $(t, t)$. (Think of it like a bridge collapsing because you passed a truck through it, or a use once only connection.) Now that we are at $t$, our frame has changed from $\mathbb{M}$ to $\mathbf{N}$. We evaluate $A$ at $t$ but the accessibility has changed, it is now the frame $\mathbf{N}$ not the frame $\mathbb{M}$.

Figure 7 shows the available paths.

The reactive evaluation at the fixed frame $\mathbb{M}$ does give rise to a set of theorems $\mathbb{L}(\mathbb{M})$. This set may be axiomatised as a Hilbert or Gentzen system or may be

Fig. 7 Available paths

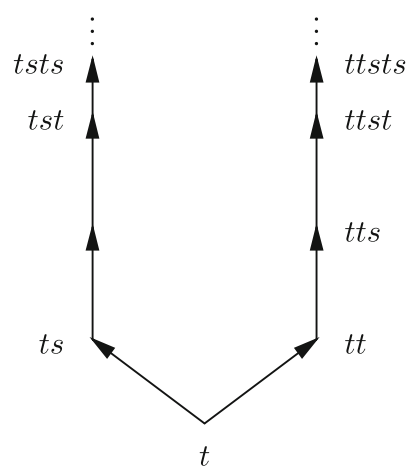


characterised by a tableaux system. Since any tableaux system follows closely the semantic evaluation procedures, this leads us to the idea of Reactive Tableaux.

The next section will define abstractly the notion of a reactive tableaux. In the rest of this section we need to show what kind of logic we get from the frame $\mathbb{M}$ and what kind of tableaux system corresponds to it.

First let us axiomatise the logics of frame $\mathbf{F}_{2}$ and $\mathbb{M}$ and show that the logic of $\mathbb{M}$ cannot be characterised by any class of ordinary frames.

Remark 1.17 (Methodological comments) We saw that reactive evaluation in the frame $\mathbb{M}$ moved also to evaluation in the frame $\mathbf{N}$. This is typical. In general, since reactive evaluation in a reactive frame $\mathbb{N}$ changes the frame as we proceed in the evaluation, we end up with a complex interaction of evaluations in some traditional frames $\mathbf{N}_{1}, \ldots, \mathbf{N}_{k}$.

This has implications to how we axiomatise and develop tableaux for the logic of $\mathbb{N}$. We need to understand the logics and tableaux of the traditional frames $\mathbf{N}_{1}, \ldots, \mathbf{N}_{k}$.

We see that in principle we need a mutually recursive axiomatisation of several logics, all done together. This is already a new kind of game. We take several logics $\mathbb{L}_{i}$ and recursively define a new combination of them, where the exact way they are combined is dictated and guided by the reactivity involved.

This observation may necessitate different axiomatic formulations of $\mathbb{L}_{i}$, not necessarily the traditional ones, in order to facilitate their combination.

The next example shows how we need to reformulate the traditional modal $\mathbf{K}$, in order to axiomatise the logic of $\mathbb{M}$.

We also see, by the way, that turning a logic reactive is a very special form of combining logics.

Definition 1.18 (Modal $\mathbf{K}$ without necessitation) Consider the following formulation of modal logic $\mathbf{K}$, without the necessitation rule. ${ }^{2}$

1. (a) All substitution instances of truth functional tautologies $A$.

(b) If $A$ is a substitution instance of a truth functional tautology then $\square^{m} A, m \geq 1$ is an axiom.

2. (a) All substitution instances of $(\square(A \rightarrow B) \rightarrow(\square A \rightarrow \square B))$

(b) All substitution instances of $\square^{m}(\square(A \rightarrow B) \rightarrow(\square A \rightarrow \square B)$ ) for any $m \geq 1$.

3. Note that the necessitation rule

$$
\frac{\vdash A}{\vdash \square A}
$$

is derivable now for this formulation of $\mathbf{K}$.

\footnotetext{
${ }^{2}$ We need this complicated axiomatisation of $\mathbf{K}$ so that it becomes easy to axiomatise the nonnormal logic of the frame $\mathbf{F}_{2}$. It also becomes easy to transform this axiomatisation into a reactive axiomatisation of the logic of the frame $\mathbb{M}$.
} 
Definition 1.19 (The logic of the frame $\mathbf{N}$ ) The logic of the frame $\mathbf{N}$ of Fig. 6 is easy to axiomatise. It is a normal logic. We can take the usual axioms of $\mathbf{K}$ with necessitation and add very simple axioms for $\diamond$, as follows.

1. All substitution instances of truth functional tautologies.

2. $\square(A \rightarrow B) \rightarrow(\square A \rightarrow \square B)$

3. $\frac{\vdash A}{-\square A}$

4. $\frac{\vdash A \quad \vdash A \rightarrow B}{\vdash B}$

5. $\sim \diamond A \leftrightarrow \diamond \sim A$

6. $\diamond \diamond A \leftrightarrow A$.

Theorem 1.20 The logic of Definition 1.19 is complete for the intended semantics of the frame $\mathbf{N}$ of Fig. 6.

Proof Easy.

Definition 1.21 (The logic $\mathbb{L}\left(\mathbf{F}_{2}\right)$ ) Consider the following modal axiom schemata, added to the axioms of modal logic $\mathbf{K}$ in the formulation without necessitation, as presented in Definition 1.18.

1. $A \rightarrow \diamond A$

2. (a) $\sim A \wedge \diamond A \rightarrow \square(A \wedge \diamond \sim B \rightarrow \sim \diamond B)$

(b) $\sim A \wedge \diamond A \wedge \sim B \wedge \diamond B \rightarrow \diamond(A \wedge B)$

(c) $\sim A \wedge \triangleright(A \wedge B) \rightarrow \square(A \rightarrow B)$

3. $A \rightarrow \square \diamond A$

4. Note that since $\mathbf{K}$ was formulated without necessitation we do not have the axiom $\square(A \rightarrow \diamond A)$.

The logic is not normal and its theorems are what holds in the actual world only.

5. We define the notion of $\Delta \vdash_{\mathbb{L}_{\left(\mathbf{F}_{2}\right)}} A$ in this logic to be $\Delta \cup \mathbf{A x i o m s} \vdash_{\mathbf{K}} A$, where Axioms is the set of all substitution instances of the axioms (1)-(3) above.

6. $\Delta$ is a theory of the logic if $\Delta \supseteq$ Axioms. It is consistent if it is consistent in $\mathbf{K}$.

Lemma 1.22 The logic $\mathbb{L}\left(\mathbf{F}_{2}\right)$ of Definition 1.21 is complete for the frame $\mathbf{F}_{2}$.

Proof We start by showing soundness. We check each axiom, they all must hold at $t$.

1. holds because $t R t$ holds

2. (a) Assume $t \vDash \sim A \wedge \diamond A$. This means $s \vDash A$. We show that $t \vDash \square(A \wedge \diamond \sim$ $B \rightarrow \sim \diamond B$ ). The only possible world accessible to $t$ (out of $\{t, s\}$ ) in which $A$ can hold is $s$. So if $s \vDash \diamond \sim B$, this means $t \vDash \sim B$ and hence $s \vDash \sim \diamond B$.

(b) Assume $t \vDash \sim A \wedge \diamond A \wedge \sim B \wedge \diamond B$. The only way this can hold at $t$ is that $s \vDash A \wedge B$. So $t \vDash \diamond(A \wedge B)$.

(c) Assume $t \vDash \sim A \wedge \triangleright(A \wedge B)$. This means $s \vDash B$. Hence $t \vDash \square(A \rightarrow B)$ because the only accessible world to $t$ in which $A$ holds is $s$ and $s \vDash B$.

3. Follows from the fact that in the frame $R$ is symmetric.

To prove completeness we show every consistent theory has a model with frame $\mathbf{F}_{2}$. Let $\Delta$ be a complete and consistent theory of the logic of Definition 1.21. We can 
assume that for some wff $\alpha_{0}$ we have $\sim \alpha_{0} \wedge \diamond \alpha_{0} \in \Delta$. If no such $\alpha$ exists then for all $\alpha, \sim(\sim \alpha \wedge \diamond \alpha) \in \Delta$, i.e., $\alpha \vee \sim \diamond \alpha \in \Delta$, which is $\diamond \alpha \rightarrow \alpha \in \Delta$. Since $\alpha \rightarrow \diamond \alpha$ is an axiom we get that $\alpha \leftrightarrow \diamond \alpha \in \Delta$ for all $\alpha$. This means $\Delta$ is essentially a consistent classical theory and can be given a one point classical model.

So let us assume that for some $\alpha_{0}, \sim \alpha_{0} \wedge \triangleright \alpha_{0} \in \Delta$, and find a model for $\Delta$.

Let $\Theta_{0}=\{E \mid \sim E \wedge \diamond E \in \Delta\} \cup\{D \mid \square D \in \Delta\}$. We claim $\Theta_{0}$ is consistent in $\mathbf{K}$. Otherwise for some $D_{1}, \ldots, D_{m}, E_{1}, \ldots, E_{k}$ in $\Theta_{0}$ we have

$$
\mathbf{K} \vdash \bigwedge_{j} D_{j} \rightarrow \sim \bigwedge_{i} E_{i}
$$

where $\square D_{j} \in \Delta, \sim E_{i} \wedge \diamond E_{i} \in \Delta$, and so

$$
\mathbf{K} \vdash \square\left(\bigwedge_{j} D_{j} \rightarrow \sim \bigwedge_{i} E_{i}\right)
$$

and by pushing $\square$ through we get

$$
\mathbf{K} \vdash \bigwedge_{j} \square D_{j} \rightarrow \sim \diamond \bigwedge_{i} E_{i}
$$

We now obtain a contradiction.

Since $\sim E_{1} \wedge \diamond E_{1} \wedge \sim E_{2} \wedge \diamond E_{2}$ are in $\Delta$, then so is $\nabla\left(E_{1} \wedge E_{2}\right)$. Therefore also $\sim\left(E_{1} \wedge E_{2}\right) \wedge \diamond\left(E_{1} \wedge E_{2}\right) \wedge \sim E_{3} \wedge \diamond\left(E_{3}\right) \in \Delta$ and hence $\diamond\left(E_{1} \wedge E_{2} \wedge E_{3}\right) \in \Delta$. Continuing in this manner we get $\diamond \bigwedge_{i} E_{i} \in \Delta$, a contradiction since $\Delta$ is consistent and $\mathbf{K} \vdash \bigwedge_{j} \square D_{j} \rightarrow \sim \diamond \bigwedge_{i} E_{i}$ and $\bigwedge_{j} \square D_{j} \in \Delta$ and so $\sim \diamond \bigwedge_{i} E_{i} \in \Delta$.

Therefore $\Theta_{0}$ is consistent and can be extended in $\mathbf{K}$ to a complete theory $\Theta$. We now show that if $A \in \Delta$ then $\diamond A \in \Theta$. This holds because $A \rightarrow \square \diamond A \in \Delta$. We also have that if $\square A \in \Delta$ then $A \in \Delta$. This follows form the axiom $A \rightarrow \diamond A$. We are now ready to construct a model for $\Delta$.

Let $S=\{\Delta, \Theta\}$. We know $\Delta \neq \Theta$ because $\sim \alpha_{0} \in \Delta$ and $\alpha_{0} \in \Theta$. Let $R$ be defined as $R=\{(\Delta, \Delta),(\Delta, \Theta),(\Theta, \Delta)\}$.

Let the actual world be $\Delta$. Let $h(q)=\{X \in S \mid q \in X\}$.

We claim for any $X, A$ we have

(*) $\quad X \vDash A$ iff $A \in X$.

We need to check only the cases of $\square A \in \Delta$ and $\square A \in \Theta$.

Assume $\square A \in \Delta$. Then $A \in \Delta$. Otherwise $\sim A \in \Delta$ and hence $\diamond \sim A \in \Delta$, a contradiction.

Now assume $\sim \square A \in \Delta$. Then if $\sim A \in \Delta$, we are finished. If $A \in \Delta$, we get $A \wedge$ $\diamond \sim A \in \Delta$ then by construction $\sim A \in \Theta$ and we are finished.

Now assume $\square A \in \Theta$. We need to show $A \in \Delta$. Otherwise $\sim A \in \Delta$ and hence $\square \diamond \sim A \in \Delta$ and hence $\diamond \sim A \in \Theta$ a contradiction.

Assume now that $\diamond \sim A \in \Theta$. We need to show $\sim A \in \Delta$ If $A \in \Delta$ we get $\square \nabla A \in$ $\Delta$ hence $\diamond A \in \Theta$. But $\sim \alpha \wedge \diamond \alpha \rightarrow \square(\alpha \rightarrow(\diamond \sim A \rightarrow \sim \diamond A))$ holds and we get a contradiction.

This completes the proof of Lemma 1.22. 
Lemma 1.23 (Axioms for $\mathbb{L}(\mathbb{M})$ ) The following schemas hold in the reactive frame $\mathbb{M}$ under any assignment.

(a) The following axioms

(1*) • $A \rightarrow \square \diamond A$

- $A \rightarrow \diamond A$

For any theorem $A$ of the logic of the frame $\mathbf{N}$ of Definition 1.19.

(2*) Same as axiom (2) of Definition 1.21.

(3*) $\quad A \wedge \diamond \sim A \rightarrow \square(\sim A \rightarrow \diamond A)$.

(b) $\sim A \wedge \diamond A \rightarrow \diamond(\sim A \wedge \square A)$

(c) If $x$ is an atom then there exists a model for $\sim x \wedge \diamond x$.

Proof Axiom group (a) hold in the frame $\mathbb{M}$ for similar reasons as checked in the soundness part of Lemma 1.22.

We now check (b).

Assume $t \vDash \sim A \wedge \diamond A$. This implies $t \vDash \sim A$ and $s \vDash A$. We now show $t \vDash \diamond(\sim$ $A \wedge \square A$ ). We use the fact that $t R t$ holds. We go to $t$, to check whether $t \vDash \sim A \wedge \square A$. Indeed, $t \vDash \sim A$. But now that we have passed through the connection $t R t$, because of reactivity, $t R t$ no longer holds, it was disconnected. The only possible world accessible to $t$ is $s$, and $s \vDash A$ hence $t \vDash \square A$. See Definition 1.2, item 2(b).

(c) Clearly holds

Lemma 1.24 (Reactive frames are stronger than ordinary frames) The logic of the reactive frame $\mathbb{M}$ is not complete for any class of ordinary Kripke frames.

Proof Suppose there is a class $\mathbb{K}$ of ordinary frames for which $\mathbb{L}(\mathbb{M})$ is complete. This means that every instance of axioms (a), (b) of Lemma 1.23 holds at the actual world for any assignment $h$ into any of the frames in $\mathbb{K}$. We also know that there exists a frame $(S, R, t)$ and an assignment $h$ such that (c) of Lemma 1.23 holds at $t$.

We shall use the above to derive a contradiction. We start with any frame $(S, R, t)$ in which every instance of (a) of Lemma 1.23 hold under any $h$ and see what are its properties. We prove (1), (2), (3) below:

$$
t R s_{1} \wedge t R s_{2} \wedge t \neq s, \wedge t \neq s_{2} \Rightarrow s_{1}=s_{2}
$$

Assume otherwise.

Let $\sim x$ hold at $t$ and $x$ hold at $s_{1}$ and $s_{2}$ and let $u$ hold at $s_{1}$ and $\sim u$ hold at $t$ and $s_{2}$. Figure 8 displays this situation.

Fig. 8 Illustrating Lemma 1.24

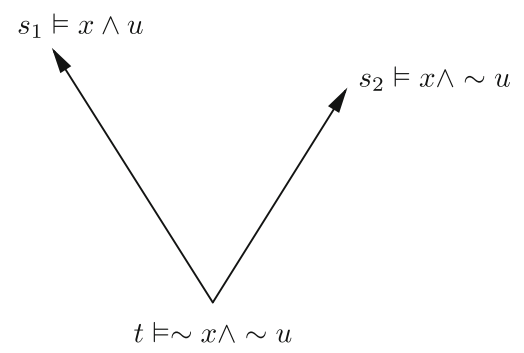


Let $\alpha=x \vee u$.

Therefore by the axiom

$$
\sim \alpha \wedge \diamond(\alpha \wedge u) \rightarrow \square(\alpha \rightarrow u),
$$

we have

$$
t \vDash \sim \alpha \wedge \diamond(\alpha \wedge u)
$$

and so $t \vDash \square(\alpha \rightarrow u)$ and so $s_{2} \vDash u$, a contradiction.

(b) We claim that $t R t$ holds. This follows from the axiom $A \rightarrow \diamond A$, for otherwise we can let $t \vDash x$ and $\forall s(t \neq x \Rightarrow s \vDash \sim x)$ and get a contradiction.

(c) We claim that $t R s \rightarrow s R t$. This is because of the axiom $A \rightarrow \square \diamond A$.

For otherwise choose an atom $x$ and let $t \vDash_{h} x$ and for all $y, s R y \Rightarrow y \vDash_{h} \sim x$. Since $s R t$ does not hold, this is OK. Then we have $t \vDash_{h} x$ but $t \forall_{h} \square \diamond x$, since at $s$ we have $s \vDash_{h} \square \sim X$.

The above (a)-(c) hold for any frame in $\mathbb{K}$.

4. We now get a contradiction. There exists a model $(S, R, t, h)$ in $\mathbb{K}$ in which (c) of Lemma 1.23 holds. Namely $t \vDash_{h} \sim x \wedge \diamond x$ holds in the model. Let $s_{1}$ be such $t R s_{1}$ and $s_{1} \vDash_{h} X$. So we must have also $t \vDash_{h} \diamond(\sim x \wedge \square x)$ from the axiom. We show that this is impossible. For let $s_{2}$ be such that $t R s_{2}$ and $s_{2} \vDash_{h} \sim x \wedge \square x$. We have shown that in any frame of $\mathbb{K}, t R s_{1} \wedge t R s_{2} \wedge t \neq s_{1} \wedge t \neq s_{2} \Rightarrow s_{1}=s_{2}$.

We have according to our data that $t \neq s_{1}$ and $s_{1} \neq s_{2}$ and $t R s_{1}$ and $t R s_{2}$. Therefore we must have $t=s_{2}$.

But $t R t$ holds and $t=s_{2} \vDash_{h} \sim x \wedge \square x$.

A contradiction.

Example 1.25 (Tableaux for the logics of $\mathbf{F}_{2}$ and of $\mathbb{M}$ ) Let us do tableaux for the logics of $\mathbf{F}_{2}$ and of $\mathbb{M}$. We know that $\sim u \wedge \diamond u \rightarrow \diamond \square u$ is refutable in the logic of $\mathbf{F}_{2}$ but is a theorem of $\mathbb{L}(\mathbb{M})$. So if we do tableaux for both we will see where the difference lies.

These two logics depend on frames with two possible worlds in them and only one reactive arrow. So our tableaux will take advantage of these special features and have the form

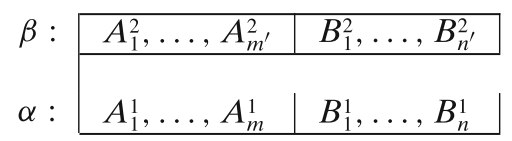

$\alpha, b$ eta are labels from $\{t, t t, s\}, \alpha$ can be $t$ or $t t$ and $\beta$ is $s$.

A tableau is closed if

1. Some $A_{i}^{k}=B_{j}^{k}$

2. $\perp$ is in the left

3. $\top$ is in the right.

The following are the rules. They are especially tailored for this particular case.

For the formulas of the form $A=\sim X$ or $A=X \wedge Y$, the left rule and the right rule for a box in row $\alpha$ or $\beta$ are the same as in classical logic. We replace the same way. The difference comes for the case of $\diamond$. 
Again we stress that we are required to do all classical connectives first for each label until we end up with only formulas which are either atomic or of the form $\diamond X$. Only at this stage do we handle $\diamond$.

In the reactive case of $\mathbb{M}$ all cases of $\diamond X_{i}$ are done simultaneously in parallel.

This is a special feature of this logic because it has at most one reactive arrow. The general case is more complex. See Section 2.

\section{$\diamond$ rule for $\alpha$}

Let $\diamond X_{i}, i \in I$ be on the left and $\diamond Y_{j}, j \in J$ be on the right. Let $I_{1}$ be any subset of $I$.

Replace

$$
\begin{array}{c|c|c|}
\beta= & A_{i}^{2} & B_{j}^{2} \\
\cline { 2 - 3 } & A_{i}^{1} & B_{j}^{1} \\
& \diamond X_{i} & \diamond Y_{j} \\
\hline
\end{array}
$$

By some of the following, for all $I_{1}$

$$
\begin{array}{rl|l|l|}
\tau_{I_{1}}=\beta: & A_{i}^{2} & B_{j}^{2} \\
& X_{I, i \in I-I_{1}}: & Y_{j, j \in J} \\
\cline { 2 - 3 } & A_{i}^{1} & B_{j}^{1} \\
X_{i}, i \in I & Y_{j}^{1}, j \in J \\
\hline
\end{array}
$$

$I_{1} \neq \varnothing$.

$$
\begin{array}{rl|l|}
\tau_{\varnothing}=\beta: & A_{i}^{2} & B_{j}^{2} \\
& X_{i}, i \in I & Y_{j}, j \in J \\
\cline { 2 - 3 } \alpha_{2}: & A_{i}^{1} & B_{j}^{1} \\
\cline { 2 - 3 } &
\end{array}
$$

As follows.

\section{Case $\mathbb{M}$}

- If $\alpha=t$ then $\alpha_{1}=t t$ and $\tau$ is replaced by all possible $\tau_{I_{1}}, I_{1} \subseteq \subseteq_{I_{1} \neq \varnothing} I$.

- If $\alpha=t t$, then $\alpha_{2}=t t$ and $\tau$ is replaced by $\tau_{\varnothing}$

- If $\alpha=t$ and $I=\varnothing$ then $\alpha_{2}=t$.

Case $\mathbf{F}_{2}$

In this case $\alpha=\alpha_{1}=\alpha_{2}=t$ and $\tau$ is replaced by all of $\tau_{I_{1}}$, for all $I_{1} \subseteq I$.

$\diamond$ rule for $\beta$

Replace

$$
\begin{array}{rl|l|l|}
\cline { 2 - 3 } \tau=\beta: & A_{i}^{2} & B_{j}^{2} \\
& \diamond X_{i}, I \in I & \diamond Y_{j}, j \in J \\
\cline { 2 - 3 } & A_{i}^{1} & B_{j}^{1} \\
\hline
\end{array}
$$


by

$$
\begin{array}{c|l|l|}
\tau=\beta: & A_{i}^{2} & B_{j}^{2} \\
\cline { 2 - 3 } \alpha: & A_{i}^{1}, X_{i} I \in I & B_{j}^{1}, Y_{j}, j \in J \\
\hline
\end{array}
$$

This is done for any of the logics and any $\alpha, \beta$.

Example 1.26 Consider the tableau $\tau_{0}$ below.

$$
\begin{array}{l|l|l|}
\tau_{0}=s: & & \\
\cline { 2 - 3 } t: & \sim u & \\
& & \diamond \square u \\
\hline & \nabla u & \\
\hline
\end{array}
$$

Let us operate on it using the rules of $\mathbf{F}_{2}$ and in parallel the rules of $\mathbb{M}$. The tableau should close for $\mathbb{M}$ and give a countermodel for $\mathbf{F}_{2}$.

The following sequence of replacements is what we get

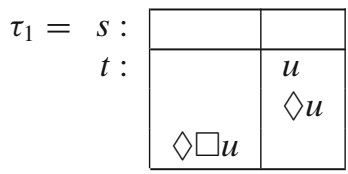

Here we should be doing $\diamond u$ and $\diamond \square u$ simultaneously, but $\diamond u$ cannot go to $\alpha$ because $u$ is false at $\alpha$ so we can do it first!

$$
\begin{array}{c|l|l|}
\tau_{2}=s: & \mathrm{u} & \\
t: & & u \\
& & \\
& & \\
\end{array}
$$

We got from $\tau_{1}$ to $\tau_{2}$ using the $\diamond$ rule for $\alpha$. This rule gives two options, but one of them is a closed tableau in this case. So we took only one of the options. We have to use now the right $\diamond$ rule for $\alpha$, for the formula $\diamond \square u$. We get the tableau $\tau_{3}$ with $\alpha_{3}$.

$$
\begin{array}{rl|l|}
\tau_{3}=s: & u & \square u \\
\alpha_{3}: & & u \quad \square u \\
\hline
\end{array}
$$

For logic $\mathbf{F}_{2}, \alpha_{3}=t$. For the logic of $\mathbb{M}, \alpha_{3}=t t$.

Using right negation rule we get $\tau_{4}$.

$$
\begin{array}{c|l|l|}
\tau_{4}= & s: & u \\
& \diamond \sim u & \\
\alpha_{3}: & \diamond \sim u & u \\
\hline
\end{array}
$$

We continue by looking at the left $\diamond$ rule for $s$, which yields:

$$
\begin{array}{c|l|l|l|}
\tau_{5}= & s: & \mathrm{u} & \\
\cline { 3 - 4 } & \alpha_{3} & \diamond \sim u & u \\
\cline { 3 - 4 } & &
\end{array}
$$


In the logic $\mathbf{F}_{2}, \alpha_{3}=t$ and so we replace $\tau_{5}$ by two tableaux, one of them has $u$ at the right hand side of the $\alpha_{3}=t$ row. This is tableau $\tau_{6}$ which gives us a countermodel. The other is $\tau_{7}$ which is closed. For the case of $\mathbb{M}, \alpha_{3}=t t$ and the only option is to replace $\tau_{5}$ by $\tau_{7}$ which is closed.

$$
\begin{aligned}
& \begin{array}{r|l|l|}
\tau_{6}=s & u & \\
\cline { 2 - 3 } \alpha_{3}=t: & & u \\
\cline { 2 - 3 } & &
\end{array}
\end{aligned}
$$

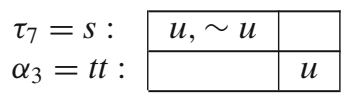

\section{Reactive Beth tableaux}

We now discuss reactive Beth tableaux. To deal with that we need a general discussion of tableaux for modal logic and its relationship to modal labelled deductive systems.

Let $\mathbb{K}$ be a set of frames of any kind, ordinary frames or reactive frames. There are two main ways of presenting $\mathbb{K}$.

1. List the members of $\mathbb{K}$ or generate them inductively.

2. Use a meta-predicate $\Psi$ in some language capable of talking about properties of frames and letting

$$
\mathbb{K}=\{\mathbb{F} \mid \Psi(\mathbb{F}) \text { holds }\}
$$

The way we develop tableaux for $\mathbb{L}(\mathbb{K})$ depends on the way $\mathbb{K}$ is presented.

Suppose $\mathbb{K}$ is presented as a set of specific finite frames, namely $\mathbb{K}=$ $\left\{\mathbb{F}_{1}, \mathbb{F}_{2}, \ldots,\right\}$, where $\mathbb{F}_{n}$ are all finite. We can do tableaux for each $\mathbb{F}_{i}$ much in the same spirit as we did in Section 1 and prove a completeness theorem of the form:

$\mathbb{L}(\mathbb{K}) \vdash A$ iff for each $\mathbb{F}_{i}$, the $A$ tableaux in $\mathbb{F}_{i}$ is closed.

The problem is more difficult when $\mathbb{K}$ is defined using a predicate $\Psi$. It is not simple even in the case of traditional Kripke frames with ordinary $R \subseteq S \times S$ and $\Psi$ a condition on $R$. Take for example the logic $\mathbf{K}+\diamond \square A \vee \diamond \square \sim A$. This logic is complete for a class of frame defined by a second order condition on $R$.

Let $E(x, y)=\exists a(x R a \wedge a R y)$. Then the condition is

$$
\Psi(R) \equiv \forall x \forall T[(y \in T \rightarrow E(x, y)) \rightarrow \exists a(\forall z(a R z \rightarrow z \in T) \vee \forall z(a R z \rightarrow \sim z \in T)) .
$$

Another logic is provability logic, being

$$
K+\{\diamond \diamond A \rightarrow \diamond A\}+\{\diamond A \rightarrow \diamond(A \wedge \square \sim A)\} .
$$

Provability logic is complete for the second order condition saying that every ascending $R$ sequence of points has a last element.

To do tableaux for these logics we have to creatively make something tailor made. If we take the frames for these logics and turn them reactive then we need to know 
how to modify the tableaux systems for the logics and turn them reactive. This is not systematic. We therefore propose to solve the following problem:

Reactive tableaux problem Given a tableaux system for some logic (never mind how we got it or what that logic is), how do we make it reactive? What are the features we change in it to make it reactive?

In the semantical case of Kripke models we know how to turn them reactive; we add the higher level arrows and allow the model to change as in Definition 1.8. We now ask how to do it in the syntactic case. Doing tableaux for reactive Kripke models gives us some clues. We make the tableaux dependent on the path of the use of $\diamond$ rules, by using labels.

The story, however, is much more complex than that, as the examples below will show.

The following series of Definitions intends to follow the way a reactive frame $\mathbb{F}_{t}$, with actual world $t$, changes in the course of evaluation along a path $\alpha$ into a new frame $\mathbb{F}_{\alpha}$. These concepts will help us formulate a recursive tableaux algorithm for the logic of the frame $\mathbb{F}_{t}$.

Definition 2.1 (How reactive frames change)

1. Let $\mathbb{F}_{t}=(S, \mathbb{R}, \mathbf{a}, t)$ be a switch reactive frame.

2. Let $\alpha=\left(t, t_{1}, \ldots, t_{n}\right), t_{i} \in S$ be a sequence of worlds. Let $\mathbf{e}(\alpha)=t_{n}$. We define $\mathbb{R}_{\alpha}=\left(S, \mathbb{F}, \mathbf{a}_{\alpha}, \mathbf{e}(\alpha)\right)$ as follows:

(a) For $\alpha=(t)$, let $\mathbb{F}_{\alpha}$ be $\mathbb{F}_{t}$ as in (1).

(b) Assume $\mathbb{F}_{\beta}$ is defined for $\beta=\left(t, t_{1}, \ldots, t_{n}\right)$ and let $\left(t_{n}, t_{n+1}\right) \in \mathbb{R}$ be such that $\mathbf{a}_{\beta}\left(t_{n}, t_{n+1}\right)=1$, i.e., $t_{n} \rightarrow t_{n+1}$ is active in $\mathbb{F}_{\beta}$. Then let $\mathbb{F}_{\left(\beta, t_{n+1}\right)}=$ $\left(S, \mathbb{R}, \mathbf{a}_{\beta,\left(t_{n}, t_{n+1}\right)}\right)$, (see Definition 1.8, item 2).

Definition 2.2 (Reactive tableaux) A tableau has the form $\left(\tau, \mathbb{F}_{\alpha}\right)$, where $\mathbb{F}_{\alpha}$ is as in Definition 2.1 and $\tau$ is a function on $S$ giving a pair of sets of formulas $\mathbf{L}^{\tau}(x)$ (left part of the tableau at $x$ ) and $\mathbf{R}^{\tau}(x)$, (right part of the tableau at $x$ ) for each $x \in S$. The formulas in $\mathbf{L}^{\tau}(x), \mathbf{R}^{\tau}(x)$ are either atomic or of the form $\diamond A$.

Definition 2.3 (Tableaux complexity) let $\left(\tau, \mathbb{F}_{\alpha}\right)$ be a tableau. The complexity of $\left(\tau, \mathbb{F}_{\alpha}\right)$ is a pair of numbers $(m, n)$ where $m$ is the maximal number of nested $\diamond$ in formulas of $\tau$ and $n$ is the number of formulas with maximal number $m$. So $m \geq 0, n \geq 1$. Note that our tableaux rules will reduce complexity.

Definition 2.4 (Tableaux feasibility) Let $\left(\tau, \mathbb{F}_{\alpha}\right)$ be a tableau. We perform a feasibility check on $\left(\tau, \mathbb{F}_{\alpha}\right)$ as follows.

Consider $\tau(\mathbf{e}(\alpha))$ :

$$
\mathbf{e}(\alpha): \begin{array}{|l|l|}
\hline A_{i} & B_{j} \\
\diamond X_{r} & \diamond Y_{w} \\
\hline
\end{array}
$$

$\diamond X_{r}, \diamond Y_{w}$ are all the formulas of this form in the tableaux, $r=1, \ldots, k_{1}$ and $w=$ $1, \ldots, k_{2}$. 
First observe that if $k_{1} \geq 1$ and there is no $s$ such that $(e(\alpha), s) \in \mathbb{R}$ is active then the tableau is not feasible. If there is such an $s$, then we can assume $k_{1} \geq 1$, because we can always take $X_{1}=\top$.

For each $s$ such that the arc $(\mathbf{e}(\alpha), s) \in \mathbb{R}$ is active, and for each $\nabla X_{r}$, consider the sets $\left(\mathbf{L}^{\tau, r, s}, \mathbf{R}^{\tau, r, s}\right)$ where $\mathbf{L}^{\tau, r, s}=\mathbf{L}^{\tau}(s) \cup\left\{X_{r}\right\}$ and $\mathbf{R}^{\tau, r, s}=\mathbf{R}^{\tau}(s) \cup\left\{Y_{w} \mid w=\right.$ $\left.1, \ldots, k_{2}\right\}$. Consider this pair as a classical tableaux and let $\left(\mathbf{L}_{i}^{\tau, r, s}, \mathbf{R}_{i}^{\tau, r, s}\right)$ for $i=$ $1, \ldots, k_{3}(r)$ be all open options for this tableaux. Consider the frame $\mathbb{F}_{\alpha, s}$ and let $\tau_{i}^{\alpha, s, r} i=1, \ldots, k_{3}(r)$ be the following tableaux for the frame $\mathbb{F}_{\alpha, s}$.

$$
\tau_{i}^{\alpha, s, r}(x)=\left\{\begin{array}{l}
\tau(x) \text { for } x \neq e(\alpha), s \\
\tau(x)-\diamond X_{r} \text { for } x=\mathbf{e}(\alpha), \mathbf{e}(\alpha) \neq s \\
\left(\mathbf{L}_{i}^{\tau, r, s}, \mathbf{R}_{i}^{\tau, r, s}\right) \text { for } x=s, \mathbf{e}(\alpha) \neq s \\
\left.\mathbf{L}_{i}^{\tau, r, s}-\left\{\diamond X_{r}\right\}, \mathbf{R}_{i}^{\tau, r, s}\right) \text { for } x=s=\mathbf{e}(\alpha)
\end{array}\right.
$$

Notice that the tableau $\tau_{i}^{\alpha, s, r}(x)$ has less complexity than the tableau $\tau^{\alpha}$, because we took out from it $\diamond X_{r}$. We may have added more modal sentences but they are of lower complexity.

We now continue:

We define the predicate feasible $\left(\tau, \mathbb{F}_{\alpha}\right)$ as follows:

- $\quad \operatorname{feasible}\left(\tau, \mathbb{F}_{\alpha}\right)$ iff

$$
\bigwedge_{r} \bigvee_{s} \bigvee_{i} \text { feasible }\left(\tau_{i}^{\alpha, s, r}, \mathbb{F}_{\alpha, s}\right)
$$

where $r=1, \ldots, k_{2}$

$$
s \in\{x \mid(\mathbf{e}(\alpha), x) \in \mathbb{R} \text { is active }\}
$$

and $i=1, \ldots, k_{3}(r)$.

If there are no open options for any $r$ then $\left(\tau, \mathbb{F}_{\alpha}\right)$ is not feasible. If the tableau $\tau(\mathbf{e}(\alpha))$ contains no modalities then it is feasible if it is open.

Definition 2.5 (Tableaux algorithm) The following is a recursive algorithm for a tableaux system for a frame $\mathbb{F}_{t}=(S, \mathbb{F}, a, t)$. Let us ask wether $A \models$ ? $B$.

First consider the classical tableau with $A$ on the left and $B$ on the right.

$$
c \tau: \begin{array}{|l|l|}
\hline A & B \\
\hline
\end{array}
$$

Any formula $E\left(q_{i}\right)$ of modal logic can be written uniquely as a substitution instance of a pure classical logic formula $E^{\prime}\left(q_{i}, p_{j}\right)$ with additional atoms $p_{j}$ where for the $p_{j}$ we substitute unique modal formulas $\diamond E_{j}$. Thus $E=E^{\prime}\left(q_{i}, P_{j} / \diamond E_{j}\right)$. Thus let us consider $A$ and $B$ in the tableau as classical formulas with 'atoms' which are either real atoms $q_{i}$ or 'atoms' of the form $\diamond A_{j}$ and $\diamond B_{j}$.

So we regard $c \tau$ as

$$
c \tau: \begin{array}{|l|l|}
\hline A^{\prime}\left(q_{i}, \diamond A_{j}\right) & B^{\prime}\left(q_{i}, \diamond B_{j}\right) \\
\hline
\end{array}
$$


Develop this tableau classically. There are two possibilities.

1. All its branches are closed, in which case $A \rightarrow B$ is an instance of classical tautology.

2. Some branches are open. Let $c t_{1}, \ldots, c t_{k_{0}}$ be all the open tableaux.

Let $c \tau_{\delta}$ be a fixed one of these options. Write $c \tau_{\delta}$ in the notational form

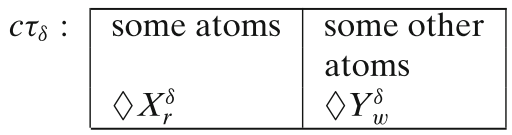

$r=1, \ldots, k_{1}, w=1, \ldots, k_{2}$

Then $\diamond X_{r}^{i}, \diamond Y_{w}^{i}$ list the modal 'atoms' which are on the left and on the right (respectively) of the tableau $c \tau_{\delta}$. We know that if we can make $\diamond X_{r}^{\delta}$ all true, $r=1,2 \ldots$ and $\diamond Y_{w}^{\delta}$ all false $w=1,2, \ldots$ then we get a countermodel.

For a fixed $\delta$, we are in the situation of Definition 2.4 , as follows. We are given $\mathbb{F}_{t}$, and we define a tableau function $\tau$ as in Definition 2.4

$$
\tau(t)=\left(\mathbf{L}^{c \tau_{\delta}}, \mathbf{R}^{c \tau_{\delta}}\right)
$$

where $\mathbf{L}^{c \tau_{\delta}}$ is the left side of $c \tau_{\delta}$ and $\mathbf{R}^{c \tau_{\delta}}$ is the right hand side of $c \tau_{\delta}$.

Let $\tau(x)=(\varnothing, \varnothing)$ for $x \neq t$. We are now exactly in the situation of Definition 2.4, with $\alpha=(t)$.

Therefore the tableau $\left(\tau, \mathbb{F}_{t}\right)$ (which is really $c \tau_{\delta}$ evaluated at $t$ ) is feasible according to Definition 2.4 iff $\bigwedge_{r} \bigvee_{s} \bigvee_{i}$ feasible $\left(\left(\tau_{i}^{t, s, r}, \mathbb{F}_{(t, s)}\right)\right)$.

We now continue the tableaux feasibility construction for each $\left(\tau_{i}^{t, s, r}, \mathbb{F}_{(t, s)}\right)$.

Note the complexity of the tableaux is reduced. We continue by induction until the tableaux we get have no $\diamond$ in them. This means they are classical tableaux and therefore are either closed or open. We can therefore know at this stage whether a countermodel exists or not.

\section{Conclusion}

We discussed reactive Kripke semantics and showed that there are modal logics which cannot be characterised by classes of ordinary Kripke frames but which can be characterised by classes of reactive frames.

We offered tableaux system for finite frame reactive Kripke models. We made essential use of the fact that the frame is finite.

Completeness theorems for ordinary arbitrary frame reactive modal $\mathrm{K}$ can be found in $[9,12]$.

Tableaux systems for ordinary reactive intuitionistic logic can be found in [8].

Acknowledgements I am grateful to Johan van Benthem and the referees for helpful criticism and useful suggestions. 


\section{References}

1. Beth, E.W.: Semantic entailment and formal derivability. Meded. K. Ned. Akad. Wet. 18(3), 309-342 (1955)

2. Crochemore, M., Gabbay, D.M.: Reactive automata. Inf. Comput. 209(4), $692-704$ (2011). doi:10.1016/j.ic.2011.01.002

3. D'Agostino, M.: Tableaux methods for classical propositional logic. In: Handbook of Tableaux Methods, pp. 45-123 (1999)

4. D’Agostino, M., Gabbay, D., Hähnle, R., Possegga, J.: Handbook of Tableau Methods. Kluwer Academic Publishers (1999)

5. Fitting, M.: Proof Methods for Modal and Intuitionistic Logics. Reidel (1983)

6. Gabbay, D.M.: Reactive Kripke semantics and arc accessibility. In: Avron, A., Dershowitz, N., Rabinovich, A. (eds.) Pillars of Computer Science*: Essays Dedicated to Boris (Boaz) Trakhtenbrot on the Occasion of His 85th Birthday. Lecture Notes in Computer Science, vol. 4800, pp. 292-341. Springer, Berlin (2008). Revised version in this issue

7. Gabbay, D.M.: Reactive Kripke models and contrary-to-duty obligations. In: van der Meyden, R., van der Torre, L. (eds.) DEON-2008: Deontic Logic in Computer Science. LNAI, vol. 5076, pp. 155-173. Springer (2008)

8. Gabbay, D.M.: Reactive intuitionistic tableaux. Synthese 179(2), 253-269 (2011). Special issue in honour of Beth, E.W., van Benthem, J., Kuipers, T., Visser, H. (eds.). http://www. springerlink.com/openurl.asp?genre=article\&id=doi:10.1007/s11229-010-9781-8

9. Gabbay, D.M.: Completeness theorems for reactive modal logics. Ann. Math. Artif. Intell. (2012). doi:10.1007/s10472-012-9315-9

10. Gabbay, D.M.: Reactive Kripke models and contrary-to-duty obligations. General theory. Journal of Applied Logic (to appear)

11. Gabbay, D.M., Schlechta, K.: Cumulativity without closure of the domain under finite unions. The Review of Symbolic Logic 1(3), 372-392 (2008)

12. Gabbay, D.M., Marcelino, S.: Modal logics of reactive frames. Stud. Log. 93, $403-444$ (2009)

13. Gabbay, D.M., Schlechta, K.: An analysis of defeasible inheritance systems. Logic Jnl. IGPL 17(1), 1-54 (2009)

14. Gabbay, D.M., Schlechta, K.: Reactive preferential structures and nonmonotonic consequence. Review of Symbolic Logic 2(2), 414-450 (2009)

15. Gabbay, D.M., Schlechta, K.: Size and logic. Review of Symbolic Logic 2(2), 396-404 (2009)

16. Gabbay, D.M., Schlechta, K.: A theory of hierarchical conditionals. J. Logic Lang. Inf. 19(1), 2-32 (2010)

17. Gabbay, D.M., Strasser, C.: Reactive standard deontic logic. J. Log. Comput. (to appear). Special issue on 60 years of deontic logic

18. Gabbay, D., Barringer, H., Woods, J.: Temporal dynamics of argumentation networks. In: Hutter, D., Stephan, W. (eds.) Volume Dedicated to Joerg Siekmann. Mechanising Mathematical Reasoning, Springer Lecture Notes in Computer Science, vol. 2605, pp. 59-98 (2005)

19. Gabbay, D.M., Barringer, H., Rydeheard, D.: Reactive grammars. In: Dershowitz, N. (ed.) A LNCS Volume in Honour of Yakov Choueka. Springer (to appear)

20. Gore, R.: Tableaux methods for modal and temporal logics. In: Handbook of Tableaux Methods, pp. 297-369 (1999) 